\title{
Las principales tendencias de la comunidad de emigrantes cubanos en España en los albores del siglo XXI
}

\section{The main trends of the community of Cuban emigrants in Spain at the dawn of the 21st century}

\author{
Jorge Luis Sosa y Addiel Pérez-Díaz \\ Instituto de Ciencia Sociales y Administración de la Universidad \\ Autónoma de Ciudad Juárez, Chihuahua, México
}

\begin{abstract}
Resumen
La comunidad de inmigrantes cubanos está basada en que son uno de los grupos de emigrantes de más antigua presencia en España, por razones de relación histórica y de la situación política de los últimos años. En el pasado, Cuba fue uno de los principales destinos de la emigración de españoles. La última etapa migratoria, que se inicia en 1959, invierte el sentido, Cuba-España. El objetivo central de este artículo es analizar los rasgos fundamentales del fenómeno migratorio cubano en España. Para ello nos apoyamos en una investigación de corte cuantitativo que revisa disímiles fuentes estadísticas.
\end{abstract}

Palabras clave: Migración cubana, tendencias, España.

Abstract

The Cuban immigrant community is based on the fact that they are one of the oldest emigrant groups in Spain, for reasons of historical relationship and the political situation of recent years. In the past, Cuba was one of the main destinations for the emigration of Spaniards. The last migratory stage, which begins in 1959, reverses the meaning, Cuba-Spain. The main objective of this article is to analyze the fundamental features of the Cuban migratory phenomenon in Spain. For them we rely on a quantitative research that reviews dissimilar statistical sources.

Key words: Cuban migration, trends, Spain. 


\section{INTRODUCCION}

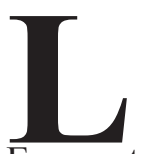

a sociedad cubana presentaba hasta en el año 2012 una tasa anual de crecimiento intercensal por cada mil habitantes negativa de -0.2. En el año 2013 fue de 3.3 y en el año 2014 fue de 2.5. En cuanto a la tasa de saldo migratorio externo por cada mil habitantes hasta el año 2012 era negativa (-4.2), comportándose por sexo de la siguiente manera: hombres $(-22,354)$ y mujeres $(-24,308) .{ }^{1}$ Sin embargo, a partir del cambio de la política migratoria en el año 2013, en que se amplió el permiso de estancia fuera del país de 11 meses a 24 meses, la tendencia cambia ligeramente. La tasa de saldo migratorio externo comenzó a ser positiva, por cada mil habitantes es de 0.3 y en el año 2014 es de 0.2 . No obstante, a partir del año $2015,{ }^{2}$ vuelve a expresarse la tendencia que ha precedido al proceso migratorio cubano después del triunfo de la revolución cubana en enero de 1959: un saldo migratorio externo negativo (ONEI, 2017).

Por otro lado, según estimaciones de la ONEI (Oficina Nacional de Estadísticas e Información de la República de Cuba) en el año 2017, el comportamiento de la estructura de población por grupos de edades hace de Cuba uno de los países más envejecidos de América Latina. Los datos estadísticos muestran un descenso paulatino de la población entre 0 a 14 años, representando 16.1 por ciento, la población que oscila entre 15 a 59 años representa 63.8 por ciento y la población de 60 años o más es 20.1 por ciento. La edad promedio de la población cubana es de 38.9 años. Recientes estudios de la entidad han confirmado que para el año 2025, la isla será el país más envejecido de la región y uno de los 25 más envejecidos en todo el mundo, en lo cual tiene un efecto directo el proceso migratorio cubano.

La comunidad de inmigrantes cubanos está basada en que son uno de los grupos de emigrantes de más antigua presencia en España, por razones de relación histórica y de la situación política de los últimos años. En el pasado, Cuba fue uno de los principales destinos de la emigración de españoles. La última etapa migratoria, que se inicia en 1959, invierte el sentido, Cuba-España, configurándose un grupo de inmigrantes con rasgos diferenciados de otras colonias, entre los que destacan: el alto número de

${ }^{1}$ La sociedad cubana hasta el año 2017 presenta, según el anuario demográfico del año 2017, una población de 11'230,142 de los cuales 5'584,885 son hombres y 5'636,175 son mujeres. Una de las características más sobresalientes del proceso migratorio cubano es que la tendencia a lo largo de la historia ha sido que emigran más mujeres que hombres.

${ }^{2}$ El saldo migratorio externo en el año 2015 fue de -2.2 en el 2016 fue de -1.5 y en el año 2017 fue de -2.3 . 
personas con doble nacionalidad, que es una migración sin retorno, con un alto nivel de cualificación profesional y en los que se entremezclan elementos políticos y económicos, como causas de la migración (García, 2012).

Como bien señala el investigador cubano Antonio Aja el año 1959 marca la modificación de los componentes migratorios tradicionales de $\mathrm{Cuba}^{3}{ }^{3}$ al cobrar un papel central, los elementos políticos y económicos motivados por la propia evolución del proceso revolucionario. La historia de este proceso se inicia desde la primera oleada migratoria después del triunfo revolucionario, integrada por aquellos cubanos vinculados directamente en el plano político, militar y también económico con la dictadura de Fulgencio Batista, que encontraron refugio en países como España y Estados Unidos, fundamentalmente, y continúa con otras cuya motivación política, económica y de reunificación familiar se presenta con matices diferenciales según etapas y flujos migratorios.

La ruptura del patrón migratorio tradicional cubano se produce por el aumento de las cifras de personas que emigran, y por los agentes sociales implicados en dicho fenómeno. Entre 1959 y hasta 1999 habían emigrado por todas las vías posibles hacia diferentes lugares del mundo, más de 1'079,000 cubanos, lo que produce una diversificación de los países receptores de emigrados cubanos (Aja, 2002). A ello se une la utilización de países puentes ${ }^{4}$ y las reales posibilidades encontradas con posterioridad por los inmigrantes para su traslado hacia territorio estadounidense.

En definitiva, para cada una de estas oleadas del flujo migratorio cubano es necesario señalar el carácter multicausal que históricamente ha tenido esta oleada migratoria, su vinculación con factores internos de la sociedad cubana, tales como las transformaciones políticas y económicas, que llevaron a la radicalización del proceso revolucionario cubano y que no fue aceptado por una parte considerable de la población cubana, la cual no vio otra salida que la emigración y, por otro lado, podemos señalar las difíciles condiciones económicas que ha enfrentado la sociedad cubana, a partir de la caída del bloque socialista a finales de la década de los ochenta del siglo pasado.

En efecto, pese a la crisis económica que vive España, la cifra de cubanos ha aumentado. Actualmente un total de 12,812 cubanos residen en

\footnotetext{
${ }^{3}$ Desde este momento Cuba dejó de ser un país atractivo para los emigrantes españoles. Muchos de ellos con el proceso de nacionalización y de confiscación de sus bienes y propiedades, se vieron obligados a regresar a España.

${ }^{4} \mathrm{~A}$ lo largo de la historia del proceso migratorio cubano España les ha servido a los cubanos para asentarse o para seguir viaje hacia Estados Unidos.
} 
España, la mitad entre los 16 y 44 años, según datos oficiales divulgados por el Instituto Nacional de Estadística de España (INE). De los emigrantes nacidos en la Isla, 67,986 tienen nacionalidad española y 56,826, permiso de residencia y trabajo. Por regiones, sobresalen Islas Canarias $(31,383)$, Madrid $(23,234)$ y Cataluña $(17,557)$.

Existen muy pocos trabajos que se centren fundamentalmente en el colectivo cubano en España; un interés que quizás queda eclipsado y mediatizado por el flujo constante de migrantes cubanos hacia Estados Unidos o también, porque desde España se han estudiado aquellos colectivos que, cuantitativamente, han captado más la atención, como es el caso de los rumanos, marroquíes, ecuatorianos o colombianos, o bien otro tipo de estudios que engloban las migraciones desde una perspectiva general.

En el presente artículo se recogen los resultados de una investigación sociológica mucho más amplia que persiguió, como interés principal, analizar las causas y consecuencias de la movilidad socio-espacial de los cubanos que deciden emigrar hacia España (Luis, 2017). Tras revisar la historia reciente de Cuba hemos podido comprobar que el hecho de migrar se ha convertido en algo cotidiano y usual para la población cubana y, por tanto, no desechable entre sus proyectos de vida cotidiana (García, 2012). De ahí que, tras interesarnos por las distintas oleadas migratorias que se han sucedido en la isla durante las últimas décadas (y que han conformado la existencia de una cultura migratoria en la sociedad cubana actual) hemos centrado nuestra atención en el flujo migratorio cubano hacia España $\mathrm{y}$, sobre todo, en los proyectos y trayectorias de los migrantes cubanos. El objetivo central de este artículo es analizar los rasgos fundamentales del fenómeno migratorio cubano en España, enfatizando en la posición de los inmigrantes en la estructura social española, así como las características de la migración cubana en España, a partir de una revisión exhaustiva de fuentes estadísticas.

\section{Apuntes teóricos para el estudio de las Migraciones Interna- CIONALES}

Las diferentes teorías desarrolladas para la comprensión de los procesos de migración internacional proponen mecanismos causales que operan en niveles de análisis ampliamente divergentes. Aunque las proposiciones, asunciones e hipótesis derivadas de cada perspectiva no son inherentemente contradictorias si conllevan implicaciones muy diferentes en la formulación de políticas adecuadas. Dependiendo de qué modelo se sostenga y bajo qué circunstancias, la ciencia social podría recomendar a los encar- 
gados de realizar tales políticas que tratasen de regular la migración internacional a través de cambios en las condiciones salariales y de empleo de los países de destino; a través de la promoción del desarrollo económico en los países de origen; estableciendo programas de seguridad social en las sociedades de salida; reduciendo la desigualdad de ingresos y mejorando la calidad de vida de las personas residentes en los lugares de origen las migraciones; mejorando los mercados de capital y los de futuros en las regiones en desarrollo; o a través de alguna combinación de estas actuaciones. También podría advertirse que todos estos programas serían infructuosos dados los imperativos estructurales impuestos a los movimientos internacionales cada vez más apartados de las relaciones económicas de los mercados.

En este sentido una de las teorías que ha tenido mayor impacto, hasta la actualidad, a la hora de trata de explicar las corrientes migratorias internacionales es la que se conoce con el nombre de teoría neoclásica, la cual fue originada en la segunda mitad del siglo XX. Esta resaltaba la relación existente entre la migración laboral (búsqueda de mejores salarios) y los procesos de desarrollo económico (Massey, 1998). De acuerdo con esta teoría y sus términos, la migración internacional tiene sus causas en la oferta y demanda de mano de obra. Es decir, los Estados con una amplia demanda de trabajo en relación con el capital tienen un equilibrio salarial bajo, mientras que los Estados con una dotación laboral limitada respecto al capital se caracterizan por salarios de altos.

Como bien señala el autor, anteriormente mencionado, este tipo de relación trae consigo que las diferencias salariales resultantes provoquen el desplazamiento de los trabajadores desde los países de salarios bajos hacia aquellos de salarios altos con la finalidad de mejorar sus condiciones de vida.

En definitiva, esta perspectiva contiene varias proposiciones que hay que tener en cuenta al momento de comprender las migraciones internacionales:

1. La migración internacional de trabajadores es causada por diferencias salariales entre países, lo cual tiene un efecto directo en la calidad de vida de las personas residentes en las sociedades de destino de las migraciones.

2. La eliminación de las diferencias salariales terminará con el movimiento de trabajadores, por lo que la migración no acontecerá ante la ausencia de estas diferencias. 
3. Los flujos internacionales de capital humano; es decir, de trabajadores altamente cualificados responden a las diferencias entre los índices de beneficios de capital humano y la mejora de las condiciones de vida, identificados por los trabajadores migrantes.

4. La forma en que los gobiernos pueden controlar los flujos migratorios es a través de la regulación o influencia en los mercados de trabajo en los países emisores o receptores.

Sin embargo, algunas de las posturas más clásicas vinculadas a estudios sociales sobre migraciones tendían a estimar que los y las migrantes, al dejar sus países de origen, dejaban atrás sus redes y bases culturales iniciando un proceso de identificación con el país de destino. Bajo esta idea, la migración estaría considerada como un proceso de desarraigo de un contexto nacional para desarrollar nuevas raíces culturales en otro (Eisenstadt, 1952 y 1954; Gordon, 1975, 1964, citados por Riaño y Ritzer, 2008). Actualmente, y contando ya con una amplia variedad de estudios dirigidos a esta temática, esta perspectiva ha dejado de parecer adecuada al considerar que los procesos migratorios no conducen necesariamente a unas rupturas tajantes con las redes de origen, incluso a pesar de la distinta ubicación espacial. Lo que se estaría produciendo es una redefinición de este tipo de relaciones. Algunos autores ponen el énfasis en recordar que no existe tal separación entre origen y destino, porque ambos están interconectados por un conjunto denso de redes sociales y económicas. La sociedad de destino está inyectada en la sociedad de origen y al revés (Goñalons et al, 2008).

Alrededor de este mismo planteamiento se gestó, a partir de los años noventa, lo que se ha denominado perspectiva transnacional o transnacionalismo presentándose como una corriente teórica emergente con la que se pretende analizar y entender, desde otro enfoque, los estudios sobre las migraciones. Sin embargo, el fenómeno transnacional, como tal, no es nuevo. En el pasado ya existían migrantes que mantenían relaciones, más o menos frecuentes, con sus comunidades de origen; aun así, se evidenciaba la falta de un enfoque teórico abierto y transfronterizo, que permitiese estudiar esa realidad. Por otra parte, los avances tecnológicos producidos en los últimos años en campos como la comunicación, los transportes o los flujos de información, han posibilitado una progresiva interconexión entre diferentes realidades locales. El abaratamiento del transporte a larga distancia y las nuevas tecnologías de comunicación e información permiten, a la persona que emigra, mantener vínculos asi- 
duos entre la sociedad de origen y la de destino o itinerancia, produciendo con ello una progresiva interconexión entre culturas, personas y lugares.

Los estudios sobre migraciones han intentado mostrar esta nueva realidad y la perspectiva transnacional aporta nueva luz en esta temática ya que entiende las migraciones como procesos complejos articuladores de espacios geopolíticos y culturales. Portes destaca que, aunque el fenómeno transnacional, en sí, no es nuevo, en la actualidad presenta las propiedades inéditas mencionadas: i) la presencia de las tecnologías de comunicación y transporte; ii) el nivel e intensidad del contacto que permiten estas tecnologías; iii) la progresiva mayor implicación de los Estados de origen de la migración para promover y controlar el transnacionalismo (Portes, 2005). Estas tres consideraciones están ayudando a entender de qué forma se configuran los proyectos y las estrategias migratorias en los estudios actuales.

Los primeros trabajos sobre el transnacionalismo fueron realizados por Basch, Glick Schiller y Szanton Blanc, entendiendo el transnacionalismo como aquel "conjunto de procesos por los cuales los inmigrantes forjan $\mathrm{y}$ sostienen redes sociales entrelazadas que vinculan sus sociedades de origen y de acogida" (Basch, Glick-Schiller y Szanton Blanc, 1994: 3). Estas antropólogas detectaron que las teorías anteriores sobre migración eran incapaces de captar los procesos sociales que se crean a través de los movimientos transnacionales. De ahí que, junto con otros autores, afrontan el reto de construir un nuevo marco analítico que sea capaz de captar los espacios multilocalmente y en los que los actores migrantes (entre ellos las mujeres, a las que había que dar una merecida importancia) no sean simples espectadores pasivos sino sujetos que actúan.

Se superaría, de este modo, las categorías tradicionales de emigrante e inmigrado (en las que sólo era posible una mirada unidireccional) y se pone de manifiesto que los migrantes actúan en un campo transnacional trascendiendo las fronteras de los Estados en los que se sitúa su origen y sus posibles destinos. Según plantea Roca en este campo se crea un espacio de relación a través de lazos económicos, afectivos, sociales y culturales donde circulan dinero, recursos, mensajes, relaciones, sentimientos e ideas. Se supera así la idea, algo simplista, de aquel individuo procedente de un país pobre que se dirige a un país rico, o de la sociedad tradicional a la moderna, para mejorar sus condiciones económicas de vida, o bien aquella visión no menos reduccionista del colectivo de trabajadores manejados y definidos en función de la división internacional del trabajo o de su acceso a los medios de producción, ampliando el campo de visión tanto a los 
procesos como a las estrategias que se ponen en marcha por las personas implicadas (Roca et al., 2008).

Desde las distintas aportaciones teóricas, actualmente esta perspectiva se ha convertido en el paradigma dominante dentro de los estudios sobre migraciones ya que plantea importantes retos de carácter epistemológico y metodológico y obliga a utilizar diversas técnicas y marcos de referencia teórico-conceptuales que muestran la interdependencia que existe entre distintos espacios geográficos. Portes nos lleva a valorar que nos encontramos ante una nueva perspectiva teórica con la que se pretende abordar, desde una visión diferente, el fenómeno migratorio (Portes, 2005). La perspectiva transnacional en las ciencias sociales ve las migraciones como procesos complejos, articuladores de los ámbitos culturales, sociales y económicos, entre comunidades e instituciones sociales distantes geográficamente. La plurilocalización de las comunidades se caracterizaría por los continuos flujos e intercambios de personas, bienes e información que surgen con y de la migración, y provocan una reproducción de los patrones culturales y formas simbólicas de sus comunidades de origen en los lugares que los acogen, a través de redes sociales y comunitarias.

Por otra parte, el vivir transnacional como lo denomina (Guarnizo, 2004), y que define como un activo y dinámico campo de intercambio social que involucra y afecta, simultáneamente, a los actores (individuos, grupos, instituciones) localizados en diferentes países, no se presentaría necesariamente como un periodo transitorio en la vida de las personas, sino que iría más allá de un periodo de tiempo determinado llegando a configurar un modo de vida relativamente estable tanto individual como familiarmente.

En efecto, este posicionamiento ofrece una nueva visión para el análisis de los mecanismos que, en los países receptores, están vinculados a la integración, el desarraigo y la cohesión de la población inmigrante.

Por su parte, Portes destaca que aunque la experiencia personal de la mayoría de emigrantes es dual y binacional, no es menos cierto que el vivir transnacional comporta, paralelamente, nuevas formas de asociación, de proyectos empresariales, de creación de espacios de interacción, de sociabilidad y de construcción de nuevas identidades híbridas, que se caracterizan por diferentes formas de neocomunitarismo que, sin embargo, se construyen de manera deslocalizada, ya que operan más allá de las fronteras nacionales, cruzándolas y trascendiéndolas (Portes, 1996a, 1996b). Así pues, queremos destacar como relevante de este nuevo enfoque que, además de analizar la migración desde los países receptores, recoge 
la necesidad de examinar los contextos de partida de los migrantes para entender las lógicas que existen en la migración.

Uno de los debates que más repercusión ha tenido dentro de la perspectiva transnacional es el que se refiere al papel de la mujer en las migraciones contemporáneas, la denominada: feminización de las migraciones. En lo que respecta a los estudios sobre género y transnacionalismo, esta perspectiva supone un avance en el análisis tanto de las migraciones en general como de las circunstancias específicas de género. El análisis de las mujeres migrantes, desde el enfoque transnacional, ayuda a descubrir la forma en la que operan estructuras de desigualdad de género de forma transterritorial. Las realidades transterritoriales son espacios en los que se pueden reproducir o reconfigurar las formas de desigualdad sobre la mujer, a la vez que son también espacios en los que es posible potenciar su emancipación y autonomía. Por lo tanto, la situación de estas mujeres puede ser mejor comprendida desde una óptica transnacional que desde un análisis clásico centrado en la sociedad de destino (Goñalons et. al., 2008: 4). Liliana Suárez, por su parte, destaca que los espacios transnacionales pueden llegar a desarrollar estrategias que ayuden a superar las desigualdades de género y conformar espacios de emancipación.

Estudios como los de Sassen (1998) parten de la centralidad del género para entender cómo se forman y consolidan parte de los procesos migratorios; o como los de Gregorio (1998), Oso (1998), Pedone (2005) y Herrera (2005) que recogen el papel central de las mujeres en las redes migratorias. Además, un número creciente de mujeres se ha sumado a la cifra de varones que encabezan familias transnacionales (Parreñas, 2001). El espacio transnacional permite, por tanto, hacer visible la transformación y la renegociación de las relaciones de género que, a menudo, influyen en el reparto de poder presente en la esfera doméstica, incluso cuando existen marcados patrones culturales tradicionales.

Por último, queremos señalar que no son muchos los estudios transnacionales que se han centrado, específicamente, en la variable género y son varios los autores que han querido hacer notar este vacío (Itzigsohn, 2000; Pessar y Mahler, 2003, citados por Goñalons et al., 2008: 14). Sin embargo, sí hay varios estudios que han evidenciado que el género marca diferencias en las posibilidades, experiencias y prácticas relacionadas con el transnacionalismo. Algunos autores, como Itzigsohn, indican la necesidad de incluir las dimensiones de la familia y el hogar para poder comprender mejor el papel de la variable género en los espacios transnacionales (Itzigsohn, 2000). Otros, como Donnecker, afirman que la migración 
femenina (en concreto la que se hace sin redes de apoyo) en algunos países es símbolo de transgresión, de autonomía y de decisión, y contribuye a demostrar que el espacio transnacional permite la transformación y la renegociación de las relaciones de género influyendo en el contexto familiar y local (Donnecker, 2005: 664, citado por Roca et al, 2008).

\section{Metodología APlicada en el estudio}

Esta investigación sobre la problemática migratoria cubana en España ha sido analizada desde hechos estadísticos y objetivos, e intentamos comprenderla a través de un proceso interpretativo en el que se combinaron, para describirla, los factores estructurales y de percepción humana que pudieran estar en los origines de este fenómeno (García, 1996).

Por otro lado, queremos destacar el uso de la metodología cuantitativa con relación a algunas de las variables utilizadas (edad, sexo, color de la piel, año de llegada a España y profesión) y al análisis documental vinculado a datos estadísticos de censos o resultados de otros estudios. Desde esta perspectiva, se llevó a cabo la consulta y la búsqueda de datos de tipo estadístico (Lehman, 1992) que permitieron, directa o indirectamente, de forma rigurosa o aproximada, establecer una mayor dimensión del fenómeno objeto de estudio en esta investigación: cuánto tiempo llevaban viviendo en España, cuántas horas trabajan semanalmente, qué tipo de trabajo realizaban, cuál era su situación laboral en España, cuál era su profesión y de qué derechos han disfrutado al regresar a Cuba.

Para ello, se llevó a cabo una búsqueda intensiva en distintas bases de datos, informes y publicaciones que nos permitieron recoger información sobre las características cuantitativas del fenómeno, como marco de referencia y contextual complementario para esta investigación (Valles, 1999).

La investigación constó con una recopilación y revisión bibliográfica vinculada a nuestro tema de estudio. La recogida de información bibliográfica nos ayudó a ir más allá de la mera descripción sociológica, situando el fenómeno migratorio en unos parámetros más amplios. Por otro lado, realizamos consultas y descargas de diferentes páginas webs e iniciamos la preparación del trabajo de campo posterior, con la elaboración y prueba de las diferentes guías de entrevista. Tras la realización de algunas entrevistas de prueba o experimentales, se elaboró una ficha-guía para recoger los aspectos principales de cada una de ellas, facilitando el trabajo posterior de análisis.

En general el apoyo de la investigación estuvo sustentado en fuentes estadísticas. Nos referimos a la que realizamos como resultado de la bús- 
queda y elaboración de datos de tipo estadístico y que nos facilitó información sobre algunos de los aspectos que forman parte de nuestro objeto de estudio: emigrados cubanos en España, evolución del flujo migratorio en los últimos años, edades y distribución en el territorio español. Son datos que obtuvimos básicamente de tres fuentes: Instituto Nacional de Estadística de España (INE), Oficina Nacional de Estadística e Información de la República de Cuba (ONEI), el Centro de Estudios de las Migraciones Internacionales de la Universidad de la Habana y el Consulado de la república de Cuba en España.

\section{Características de la migración cubana en España}

El vínculo migratorio entre Cuba y España es histórico. En el pasado, España mantuvo una poderosa corriente migratoria a Cuba, aunque posteriormente se invierte el sentido $\mathrm{y}$, en las últimas décadas, son los cubanos quiénes están emigrando hacia a España, primero utilizando este país de cruce hacia su destino final los Estados Unidos o en segundo lugar como un país para asentarse y residir en él.

Según la información disponible sobre los cubanos inscritos en las Oficinas Consulares de Cuba en España (actualizada hasta el II trimestre del año 2005), en España están inscritos 61,130 cubanos que representan 57.8 por ciento del total de inscritos en Europa $(105,848)$ y 7.9 por ciento del total mundial $(774,323)$. Son cifras oficiales ofrecidas por la DACRE del MINREX y que se han recogido en el estudio que ha realizado al respecto Consuelo Martín (Martín, 2006). El Anuario Estadístico del Instituto Nacional de Estadística de España (INE) no difiere mucho de esa cifra ya que recoge, para el mismo año 2005, que la población cubana en España ascendía a 69,048 personas nacidas en Cuba y radicadas en España. De este número, 41.3 por ciento eran de nacionalidad española $(28,547)$, mientras que la mayoría, 58.6 por ciento $(40,501)$, eran de nacionalidad no española. Si se tienen en cuenta los datos de DACRE/MINREX, a los 61,130 cubanos inscritos en los distintos consulados en España hasta junio 2005 habría que sumar alrededor de ocho mil personas que estarían sin escribir. A estas cifras habría que añadir a aquellos que se encontraban sin regularizar su situación migratoria en España.

Existen cinco oficinas consulares cubanas en España. En cifras absolutas, de mayor a menor cantidad, se ubican en: Madrid con 22,246 cubanos y cubanas inscritos; Barcelona con 16,602; Islas Canarias con 13,285; Santiago de Compostela con 5,009; y Sevilla con 3,991. Las estadísticas de la Dirección de Inmigración y Extranjería de Cuba (DIE), ofrecen 
las cifras de los Registros Continuos de emigración del país de 1995 a 2004, recogiendo también algunas de las características de la emigración cubana hacia España. De este modo, según los datos recogidos por este organismo durante este periodo, salieron de Cuba con destino a España 28,430 cubanos (la cifra ofrecida por fuentes españolas para el mismo periodo asciende a 51,572 cubanos).

Del total de salidas de Cuba hacia cualquier país de destino durante esos años (unas 285,900 personas hasta 2003), alrededor de 8.9 por ciento representa a los cubanos que salieron hacia España (Martín, 2006). Esto no garantiza que todos se hayan asentado aquí definitivamente, ya que hay quienes utilizan a España como puente para llegar a Estados Unidos.

El 49 por ciento de los emigrantes cubanos durante el año 1994 eran mujeres y, en el caso particular de los emigrantes hacia España, el número de mujeres representaba 56 por ciento. Asimismo, la distribución por sexos muestra que en el período 1995-2004 la mayoría de los cubanos que salen hacia España son mujeres, 60.8 por ciento, frente a 40.2 por ciento de hombres.

Observando la composición de este colectivo por color de piel vemos que, aunque solamente están disponibles las cifras correspondientes a las categorías migratorias de Emigración por Salida Definitiva (SD) y Permiso de Residencia en el Extranjero (PRE) (y de esta última sólo se conoce el dato de la mitad de las personas) en cifras absolutas de ambas categorías, predomina el total de personas blancas. Las fuentes cubanas que hemos consultado no recogen porcentajes concretos, pero afirman que se observa una clara distinción en cuanto a la Emigración por Salida Definitiva, quienes son fundamentalmente de piel Blanca, cambiando en el caso del Permiso de Residencia en el Extranjero (PRE), donde los de piel Mulata y Negra salen en mayor proporción. Además, relacionando el color de piel con el sexo se observa que los de piel blanca la mayoría son hombres, mientras que tanto en la categoría de mulatos como en la de negros, hay un ligero predominio de mujeres.

Con respecto al nivel de instrucción, predomina el nivel preuniversitario (34 por ciento), seguido por secundaria básica (21 por ciento), universitario (16 por ciento), primaria (12 por ciento) y tecnológico (siete por ciento). La distribución por sexos evidencia un predominio femenino en nivel medio, y del masculino tanto en nivel superior como en primaria.

En cuanto a las provincias de residencia en el momento de la salida hacia España, la mayoría procede de Ciudad de La Habana (56 por 
ciento). Según los datos de la DIE de 1995 a 2003, la pauta nacional por género, fundamentalmente femenina, en las salidas hacia España está determinada por las provincias orientales, sobre todo la provincia de Las Tunas. La proporción por género en el resto del país es equiparable, e incluso en algunas provincias hay ligeramente más hombres que mujeres.

Refiriéndonos a la situación laboral de los cubanos que salen hacia España, los datos se han considerado respecto a la ocupación que tenían en Cuba en el momento de la salida (sólo se desconoce la información de cuatro por ciento). Del total del periodo 1995-2004, se evidencian mayores proporciones entre personas sin vínculo laboral activo, distribuidas en orden descendente en: amas de casa (29 por ciento), desocupados (20 por ciento) donde hay el doble de hombres que de mujeres; y estudiantes (15 por ciento); menores (cinco por ciento); jubilados (cuatro por ciento), donde hay un ligero predominio masculino. La proporción de trabajadores (22 por ciento) constituye la quinta parte del total, predominando ligeramente los hombres sobre las mujeres.

Como se recoge en la Tabla 1, entre los que trabajaban más de la mitad son profesionales y técnicos, donde las ocupaciones más frecuentes son: médicos (12 por ciento); maestros (14 por ciento); ingenieros (12 por ciento); artistas (cuatro por ciento); músicos (tres por ciento) y técnicos de la salud (tres por ciento), entre otras. Una categoría laboral que también destaca es la de trabajadores administrativos (25 por ciento), seguida por la de obreros (14 por ciento), trabajadores por cuenta propia (ocho por ciento) y dirigentes (cinco por ciento). Resulta interesante que ya desde 1992 (según un estudio precedente), se observaba una tendencia creciente respecto a la salida de profesionales hacia España. Esa posibilidad se confirma como tendencia mayoritaria en 2004.

Tabla 1: Ocupación de los emigrantes cubanos antes de emigrar a España

\begin{tabular}{lr}
\hline Ocupación & Porcentaje \\
\hline Músicos & 3 \\
Trabajadores administrativos & 25 \\
Obreros & 14 \\
Trabajadores por cuenta propia & 8 \\
Dirigentes & 5 \\
Técnicos de salud & 3 \\
Médicos & 12 \\
Maestros & 14 \\
Ingenieros & 12 \\
\hline
\end{tabular}

Fuente: elaboración propia a partir de los datos publicados por los Consulados cubanos en España. 
De los 6,949 inscritos que son profesionales, 37 por ciento tiene profesiones de nivel medio vinculadas a la enfermería y al magisterio; mientras que 63 por ciento son de nivel superior: Médicos, Licenciados en Arte, Economía, Derecho y Arquitectos. En ambos casos, no se sabe la profesión específica de la mayoría de estas personas.

Tras revisar los datos ofrecidos por las distintas fuentes cubanas, en este apartado analizamos los datos que recogen las fuentes españolas. El Padrón municipal de 2009, registraba un total de 100,451 personas nacidas en Cuba y radicadas en España. De estos, 44 por ciento ya tienen nacionalidad española $(43,717)$.

En cuanto a la edad de la población cubana en España, destacamos que los grupos más significativos, según el Anuario Estadístico de 2010 del Instituto Nacional de Estadística de España (INE) (que considera tan sólo las cifras relativas a la población cubana no española), se localizan en el rango comprendido entre los 30-34 años ( 8,104 personas) y entre los 35-39 años (7,585), representando, ambos grupos, 33 por ciento de la población total. Si añadimos el tercer rango relevante, el que oscila entre los 40 y 44 años $(6,087)$, alcanzaríamos 46 por ciento. Se trataría, de este modo, de una población envejecida sobre todo considerando que los rangos de 0 a 4 años y de cinco a nueve años, representan tan solo 2.4 por ciento.

Geográficamente, y según los datos del Padrón de 2009, este colectivo se concentra básicamente en cuatro áreas: Canarias (23 por ciento), Comunidad de Madrid (19 por ciento), Cataluña (15 por ciento) y la Comunidad Valenciana (nueve por ciento). Si consideramos también las dos áreas que le siguen en importancia (Galicia, con siete por ciento y Andalucía con seis por ciento) podemos observar que en estas zonas se concentra 79 por ciento de la población cubana residente en España. Por ciudades, como se muestra en la Tabla 2, en las que más se concentran los cubanos es en Madrid (18,896), seguida de Las Palmas (11,825), Sta. Cruz de Tenerife $(11,626)$ y Barcelona $(11,357)$.

Tabla 2: Distribución geográfica de la población cubana en España por ciudades con mayor número de cubanos

\begin{tabular}{lr}
\hline Ciudad & Cantidad \\
\hline Las Palmas & 18,896 \\
Santa Cruz de Tenerife & 11,626 \\
Barcelona & 11,357 \\
Madrid & 11,825 \\
\hline
\end{tabular}

Fuente: elaboración propia a partir de datos del Padrón de 2009. 
Hemos considerado indagar sobre cuál ha sido la evolución de esta distribución durante el periodo comprendido entre 1999-2009. En este sentido hay que señalar que la población cubana ha experimentado cambios considerables durante la década analizada. En 1999, en Madrid residía 23 por ciento de esta población, seguida de cerca por Canarias (19 por ciento) y por Galicia en tercera posición (14 por ciento). Diez años después, en el año 2009, es Canarias la que concentra el mayor porcentaje de cubanos ubicados en España, exactamente 23 por ciento. Madrid ha pasado a ocupar un segundo lugar (con 19 por ciento del total) quedando Cataluña en el tercero ( 15 por ciento) y Galicia en el cuarto (siete por ciento).

Aunque Andalucía y Galicia han visto cómo se incrementaba considerablemente la población cubana en su territorio, han sido las Comunidades de Cataluña (pasando de 3,880 cubanos en 1999, a 15,018 en 2009), Madrid (de 7,982 a 18,896) y, sobre todo Canarias (de 6,317 a $23,451)$, las zonas del territorio español que han experimentado un incremento mayor a lo largo de la década revisada. En el caso de Canarias, la de mayor incremento, creemos que probablemente ha influido el flujo migratorio mantenido históricamente entre Canarias y Cuba, favorecido ahora en el caso de aquellos cubanos que tienen lazos de parentesco con antepasados españoles. La denominada Ley de la Memoria Histórica de 2007, ha propiciado una política migratoria que favorece la llegada a España de los descendientes (en particular, hijos y nietos), de españoles que emigraron a Latinoamérica entre 1936 y 1955.

Al comparar el total de inmigrantes cubanos en España con los trece países extracomunitarios con mayor número de inmigrantes, según el Anuario Estadístico de España de 2008, observamos que la población cubana ocupa el puesto número once. Además, respecto al total de inmigrantes procedentes de países extracomunitarios (que ascienden a 3’428,474), Cuba representaría 2.4, como se muestra en la Tabla 3.

A través de estos datos se deduce que, en España, la inmigración cubana no presenta cifras tan elevadas como la de otros grupos de migrantes, por ejemplo, el de ecuatorianos o marroquíes. Sin embargo, a partir de inicios de la década de los 90 , ha experimentado un incremento gradual muy significativo: de un total de 2,637 inmigrantes cubanos a finales de 1991 se ha pasado a 100,451 en 2009 , por lo que comprobamos que, durante este periodo, la población cubana en España se ha multiplicado por casi 38. Pensamos, por tanto, que es un fenómeno que merece especial atención. 
Tabla 3: Número de migrantes cubanos en España respecto a las trece nacionalidades no comunitarias más representativas

\begin{tabular}{lcc}
\hline País & $\begin{array}{c}\text { Número de Migrantes } \\
\text { países no comunitarios }\end{array}$ & $\begin{array}{c}\text { \% respecto al total de } \\
\text { emigrantes no comunitarios }\end{array}$ \\
\hline Marruecos & 621,295 & 18.1 \\
Ecuador & 434,673 & 12.7 \\
Colombia & 291,676 & 8.5 \\
Argentina & 272,985 & 8.0 \\
Bolivia & 200,749 & 5.9 \\
Perú & 136,958 & 4.0 \\
Venezuela & 130,630 & 3.8 \\
Brasil & 113,448 & 3.3 \\
China & 108,258 & 3.2 \\
República Dominicana & 96,672 & 2.8 \\
Cuba & 83,121 & 2.4 \\
Uruguay & 79,842 & 2.3 \\
Ucrania & 69,376 & 2.0 \\
\hline
\end{tabular}

Fuente: elaboración propia a partir de los datos del Anuario Estadístico de España de 2008.

El aumento gradual se ha mantenido desde inicios del periodo analizado hasta el momento actual, de ahí que nos inclinemos a pensar que será la tendencia que percibiremos durante los próximos años. Esta tendencia podría verse influenciada tanto por la crisis económica que todavía hoy afecta al país cubano, como por motivaciones diversas que empujan a cubanos y cubanas a tomar la decisión de salir del país. Además, se debe considerar un futuro incremento también condicionado por la aprobación de la ya mencionada Ley de la Memoria Histórica de 2007. Según los datos recogidos por Consuelo Martín, muchos de los cubanos que emigran lo hacen como descendientes de españoles. Son cubanos que salen del país con su nacionalidad española (en 2006, 41.3 por ciento, en 2007, 42.4 por ciento, en $2008,43.1$ por ciento) y esta modalidad continuará incrementándose aún más a partir de 2009, precisamente porque ahora la ley favorece a los nietos (Martín, 2006).

Es importante señalar, de cara a hacer una predicción para los próximos años, que, de los 100,451 cubanos en España en 2009, la mitad llegó a partir del año 2001; y que España sigue ocupando, todavía hoy, el segundo lugar dentro de los países de destino de la emigración cubana en el mundo, después de Estados Unidos. 
Por lo que respecta a la distribución de la población cubana por sexo, llama la atención que de las 100,451 personas que constituyen la población cubana en España, 55,169 son mujeres, frente a los 45,282 hombres, por lo que las mujeres, objeto de interés en este trabajo, representan 55 por ciento de este colectivo, y nos alertan de la tendencia a la feminización que presenta esta población. Esta feminización, como se aprecia en la Figura 1 se ha mantenido presente a lo largo de todo el periodo analizado.

Figura 1: Distribución de la población cubana por sexo durante el 2009

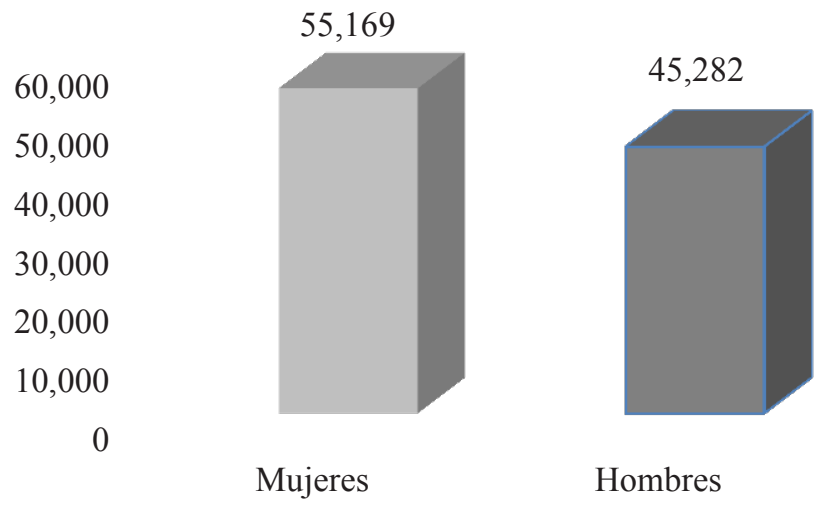

Fuente: elaboración propia a partir de los datos del INE.

Desde la Dirección de Inmigración y Extranjería de Cuba también se recoge la feminización del fenómeno migratorio cubano a España, sobre todo, con relación al resto de los movimientos de población hacia otras regiones. De forma general, destacan que la tendencia migratoria hacia España en los años 90 estaba representada mayoritariamente por hombres; sin embargo, a partir del año 2000 en adelante, se aprecia un marcado incremento de la emigración de mujeres hacia España (Martín, 2006).

Por tanto, podría estimarse que entre los inmigrantes cubanos se repite la tendencia de la feminización que está presente en otros países latinoamericanos y caribeños (García-Moreno, 2009; Pedone, 2005; Suárez, 2004). Esta tendencia general puede observarse en la Tabla 4 , en la que se aprecia el grado de representatividad de las mujeres cubanas (55.4 por ciento) respecto a los hombres cubanos en España, ocupando el séptimo lugar (junto con Venezuela) respecto a los índices recogidos para el resto de los países. 
Tabla 4: Nacionalidades de las mujeres extranjeras residentes en España, con mayor representación. Tabla comparativa 1997, 2001 y 2008

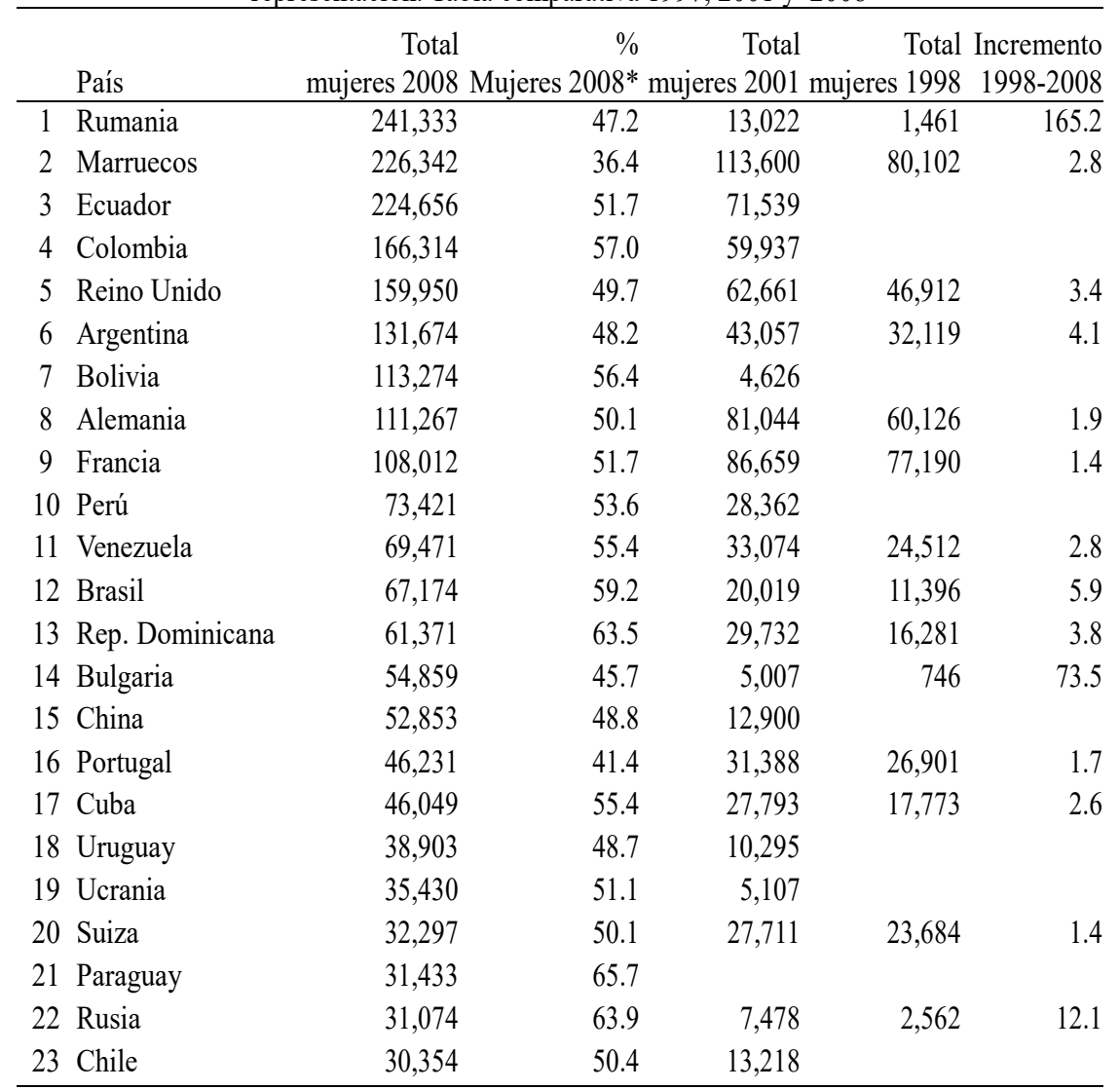

* Respecto al total de extranjeros de la misma nacionalidad.

Fuente: elaboración propia a partir de datos del Instituto Nacional de Estadística(INE).

Siguiendo con los datos recogidos en esta misma tabla, queremos destacar que, de las 23 nacionalidades con mayor representación de mujeres extranjeras en el año 2008, las mujeres cubanas ocupan la posición número 17. Cuando consideramos el incremento gradual experimentado en la cifra de mujeres de cada nacionalidad durante el periodo comprendido entre 1998 y 2008, vemos que el incremento experimentado por las mujeres cubanas representa 2.6, ocupando, con este dato, la novena posición (entre aquellos países de los que hemos encontrados datos recogidos al respecto). Comprobamos, de estos datos, que la presencia de las mujeres cubanas en España ha experimentado un incremento gradual significativo: 
de un total de 17,773 cubanas en 1998, se ha pasado a 46,049 en 2008, por lo que casi se han triplicado durante este periodo. A lo largo de este trabajo intentaremos averiguar algunas de las causas que han provocado este incremento.

Al comparar la posición que ocupan las mujeres cubanas en España respecto al resto de mujeres procedentes únicamente de países latinoamericanos, nos encontramos que, según el INE, en el año 2008 las mujeres cubanas ocupaban un noveno lugar.

En una aproximación más geográfica puede contemplarse como entre el conjunto de países que se encuentra ubicados en América Central, podemos ver que Cuba ocupa una segunda posición, superada solo por la emigración femenina procedente de la República Dominicana.

Analizamos ahora los datos relativos a la presencia de la población cubana en el mercado de trabajo español. A través de la información aportada por la Secretaría de Estado de Inmigración y Emigración, hemos revisado los datos de afiliación de los 13 países de los que, actualmente, hay un mayor número de residentes en España (entre los que se encuentra Cuba).

Por lo que respecta a la edad, la distribución de los extranjeros afiliados a la Seguridad Social por edades muestra claras diferencias con la del conjunto de los trabajadores afiliados: los extranjeros presentan rangos de edad inferiores. Sin embargo, en lo que respecta a Cuba, tiene una distribución por edades más parecida a la del conjunto de la población (junto con los argentinos y los ucranianos).

En cuanto a la distribución por categorías de la población migrante, al revisar el conjunto de los extranjeros afiliados al régimen general, observamos que el grupo más numeroso es aquel que conforma la categoría de peones en la mayoría de las nacionalidades, excepto en el caso de los cubanos, los argentinos y los chinos. En este sentido, Miguel Pajares señala que sobre todo los argentinos y los cubanos tienen una distribución por categorías que difiere bastante de la del resto de los inmigrantes, es decir, que se parece más a la de los españoles que a la de los demás grupos inmigrados (Pajares, 2009). Con esta consideración de partida, veamos ahora qué categorías laborales ocupan los cubanos en el mercado laboral español y con qué porcentajes (Tabla 5).

En la Tabla 5 podemos observar como la mayor parte de la población cubana se encuentra ubicada, en 2008, en las categorías de "Oficiales de $1^{\mathrm{a}}$ y $2^{\mathrm{a}}$ " (20.5 por ciento) y la de "Oficiales de $3^{\mathrm{a}}$ y especialistas" (19.3 por ciento). Por otra parte, llama la atención su representatividad en la 
categoría de "Ingenieros y Licenciados" (7.6 por ciento) ya que aporta el porcentaje más elevado respecto al resto de nacionalidades (seguidos de cerca solamente por los argentinos con 7.3 por ciento). Entre las once nacionalidades restantes con un mayor número de residentes en España, este porcentaje no supera 2.8 por ciento (correspondiente a Perú).

Tabla 5: Población cubana en alta laboral en la Seguridad Social en España (Régimen General y el de Minería y el Carbón). A 31 de diciembre de 2007 y 2008

\begin{tabular}{lrrr}
\hline Categoría laboral & $\begin{array}{r}\text { \% Población } \\
\text { cubana 2007 Población }\end{array}$ & $\begin{array}{r}\text { \% Mujer } \\
\text { cubana 2008 } \\
\text { cubana 2008 }\end{array}$ \\
\hline Ingenieros y licenciados & 6.3 & 7.6 & 6.9 \\
Ingenieros técnicos. Peritos & 2.3 & 2.2 & 2.4 \\
Jefes Administrativos y de taller & 1.7 & 2.2 & 1.6 \\
Ayudantes no titulados & 2.0 & 2.4 & 2.5 \\
Oficiales administrativos & 10.0 & 8.5 & 10.7 \\
Subalternos & 4.0 & 5.1 & 4.0 \\
Auxiliares Administrativos & 21.8 & 13.8 & 20.3 \\
Oficiales de 1 ${ }^{\text {a }}$ y 2 & 13.8 & 20.5 & 13.8 \\
Oficiales de 3 ${ }^{\mathrm{a}}$ y especialistas & 20.1 & 19.3 & 20.0 \\
Peones y asimilados & 18.0 & 18.4 & 17.7 \\
Total (valores absolutos) & 9,000 & 18,858 & 8,830 \\
\hline
\end{tabular}

Fuente: elaboración propia a partir de Pajares (2008 y 2009).

Comparando los datos de 2008 con los del año anterior, observamos un relevante descenso en el porcentaje de la categoría auxiliares administrativos (pasando de 21.8 por ciento en 2007 a 13.8 por ciento en 2008), e incrementándose de forma representativa los oficiales de $1^{\mathrm{a}}$ y $2^{\mathrm{a}}$ (pasando de 13.8 por ciento en 2007 a 20.5 por ciento en 2008). En cuanto a la categoría de ingenieros y licenciados, en tan sólo un año experimentó un ligero ascenso (de 6.3 pasó a 7.6 por ciento).

Prestaremos ahora atención a las Ramas de Actividad de la población cubana afiliada a la Seguridad Social, considerando ambos sexos y exclusivamente la afiliación al régimen general y al de autónomos. Como se representa en la Tabla 6. 
Tabla 6: Población cubana en alta laboral en la Seguridad Social en España (Régimen General y Autónomos). Por ramas de actividad. A 31 de diciembre de 2008

Ramas de actividad Población cubana en España

Industrias extractivas 19

Industria manufacturera 1,495

Construcción 1,876

Comercio, reparación de vehículos 4,005

Hostelería 4,689

Transporte, almacenamiento 976

Intermediación financiera

Servicios empresariales, inmobiliarias 3,326

Administración pública, seguridad social

Educación

Actividades sanitarias, servicios sociales

Otras actividades sociales, servicios personales

Total

Fuente: elaboración propia a partir de Pajares (2009).

Vemos que las actividades más desarrolladas por cubanos y cubanas son el comercio, reparación de vehículos (en la que ocupa la posición número diez respecto a los trece países de los que, actualmente, hay un mayor número de residentes en España) y la hostelería (en la que ocupa la posición número once). Por lo que respecta a construcción (importante nicho laboral de gran parte de la población inmigrada en España) aunque la cifra de afiliados cubanos es elevada respecto al resto de las ramas de actividad $(1,876)$, ocupa la última posición respecto al resto de las trece nacionalidades (con casos como los de Rumania con 59,460; Marruecos con 41,479 o Ecuador con 34,108).

Revisamos ahora, con la ayuda de la Tabla 7, el tipo de afiliación que tienen los trabajadores cubanos. Observamos que en 2008 el Régimen General destacó claramente sobre los demás con 18,858 afiliaciones frente a otros como el de empleados de hogar $(2,130)$ o el de autónomos $(1,631)$.

$\mathrm{Si}$ observamos los datos correspondientes a empleados de hogar (otro de los nichos laborales, sobre todo, con relación a las mujeres migrantes) vemos como se ha incrementado desde 2006 a 2008. Aun así, Cuba ocupa el penúltimo lugar (después de Argelia, con 430) respecto a los restantes once países (con casos como Ecuador con 28,669 afiliados, o Bolivia y Colombia con más de 20 mil). 
Tabla 7: Tipo de afiliación de los trabajadores cubanos

\begin{tabular}{lrrr}
\hline & 2006 & 2007 & 2008 \\
\hline Régimen General & 19,983 & 19,724 & 18,858 \\
Autónomos & 1,656 & 1,705 & 1,631 \\
Régimen Agrario & 270 & 326 & 397 \\
Empleados Hogar & 1,400 & 1,679 & 2,130 \\
Total & 23,309 & 23,434 & 23,016 \\
\hline
\end{tabular}

Fuente: elaboración propia a partir de Pajares (2009).

Para finalizar queremos insistir en algunos datos sobre las mujeres cubanas en España en lo que respecta a su posición en el mercado laboral español. Volvemos para ello a las cifras de afiliación de la población cubana en el régimen de Empleados de Hogar. A través de estudios recientes, se ha observado que el servicio doméstico se presenta como uno de los sectores laborales en el que se ubican la gran mayoría de mujeres inmigrantes en España, independientemente de su nivel formativo. Considerando que Cuba ocupa el penúltimo lugar de afiliaciones a este régimen, intuimos que la mujer cubana se salva de esta tendencia general. Cabrá averiguar, durante nuestro análisis cuáles han sido los inconvenientes $\mathrm{y}$ las oportunidades encontradas en su trayectoria laboral.

Según vimos antes, del total de cubanos residentes en España, el mayor porcentaje corresponde a mujeres, 55.9 por ciento. La presencia de la mujer cubana también es elevada si comparamos ambos sexos con relación a su situación de alta laboral en la Seguridad Social en España. El conjunto de cubanos y cubanas en esta situación ascendía, en 2008, a 23,167, de los cuales 48.7 por ciento eran mujeres. Este 48.7 por ciento resulta relevante si consideramos que, del total de extranjeros de todas las nacionalidades dados de alta en la Seguridad Social a 31 de diciembre de 2008, las mujeres representan 42.2 por ciento (Tabla 8).

Las categorías laborales ocupadas por las mujeres cubanas en España según datos de 2008 se recogen en la Tabla 9. Observamos que son las categorías de auxiliar administrativo (20.3 por ciento) y la de oficiales de $3^{\mathrm{a}}$ y especialistas ( 20 por ciento) las que presentan porcentajes más elevados. En el caso de ingenieros y licenciados, seguiría la misma línea que hemos destacado para el conjunto de cubanos ( 6.9 por ciento) ya que, junto con las argentinas, presentan el porcentaje más elevado respecto al resto de nacionalidades. Entre las once nacionalidades restantes con un mayor número de residentes en España, este porcentaje no supera 3.3 por ciento (correspondiente a Argelia). 
Tabla 8: Representatividad de la mujer cubana en España

\begin{tabular}{lcccc}
\hline Sexo & $\begin{array}{c}\text { Población cubana en España } \\
\text { (Padrón 2009) }\end{array}$ & \multicolumn{2}{c}{$\begin{array}{c}\text { Población cubana según alta } \\
\text { seguridad social (OPI, 2008) }\end{array}$} \\
\hline Hombres & 45,282 & $44.1 \%$ & 11,885 & $51.3 \%$ \\
Mujeres & 55,169 & $55.9 \%$ & 11,282 & $48.7 \%$ \\
Total & 100,451 & $100.0 \%$ & 23,167 & $100.0 \%$ \\
\hline
\end{tabular}

Fuente: elaboración propia a partir de datos del Padrón de 2009 y del OPI 2008.

Tabla 9: Categorías laborales que ocupan las mujeres cubanas en España, 2008

\begin{tabular}{lr}
\hline Categoría laboral & Cantidad \\
\hline Médicos & 12 \\
Maestros & 14 \\
Ingenieros & 12 \\
Artistas & 4 \\
Músicos & 3 \\
Administrativos & 25 \\
Obreros & 14 \\
Trabajadores por Cuenta Propia & 8 \\
Dirigentes & 5 \\
Técnicos de la Salud & 3 \\
\hline Fuente: elaboración propia a partir de datos de OPI 2008
\end{tabular}

Fuente: elaboración propia a partir de datos de OPI 2008

Desde un análisis más general, las marroquíes y las ecuatorianas son las que tienen porcentajes más altos en la categoría de peones y asimilados, seguidas de las argelinas, las bolivianas, las ucranianas, las búlgaras y las rumanas. En las categorías administrativas, los porcentajes más altos los tienen las cubanas, las argentinas, y las peruanas. $\mathrm{Y}$ ya en las categorías más altas sólo destacan los porcentajes de las cubanas y de las argentinas (aunque han crecido, respecto a los datos del año anterior, los porcentajes de las peruanas) (Pajares, 2009).

La cubana, como comunidad extranjera, registrada y asentada en España, resulta ser vieja. En la década de los años 30 del siglo pasado había censados en Cuba alrededor de 257,596 españoles, número que se mantuvo, con ciertas variaciones, hasta la década de los años 50. Este elemento define una de las características fundamentales de la migración cubana a España: "el alto número de nacionalizados españoles y que, por tanto, están muy integrados y asimilados en nuestra sociedad", según las investigadoras González Yanci y Aguilera Arilla (2002: 12). 
En efecto, como bien señalan las autoras, Cuba fue una de las últimas colonias españolas, de ahí que fueses uno de los principales destinos de la emigración española. De modo que el flujo migratorio es, quizás, el más constantemente mantenido entre dos territorios, variando el sentido de los desplazamientos después del triunfo de la Revolución Cubana.

La inmigración cubana no es hoy el resultado del reciente cambio experimentado en España de país emisor a país receptor de inmigrantes. Antes de que este hecho fuera una realidad, comenzó a llegar un flujo de cubanos que puso en España su meta, o su escala, en una emigración de carácter político, que iría evolucionando a lo largo del tiempo.

En esta migración hay muchas razones de sangre, como habíamos señalado anteriormente. Muchos de los ciudadanos cubanos que vienen lo hacen porque son de origen español y, a veces, han mantenido la nacionalidad de sus padres y porque, por ser en su mayoría inmigrantes de carácter político, precisan de una carta de invitación, fácil de obtener desde España, donde, en muchos casos, los cubanos cuentan con familiares. España ha supuesto para los cubanos una, y a veces la única, puerta de salida hacia el mundo occidental.

Por otro lado, podemos decir, que existe una marcada dualidad entre los miembros de la comunidad de inmigrantes cubanos que está establecida y asentada y los que llegan en los últimos años (González Yanci y Aguilera Arilla, 2002) entre los que hay una notable precariedad económica que los iguala a los inmigrantes del Tercer Mundo. Dicha dualidad, que también suele darse en otros grupos de inmigrantes, es más acentuada en el caso cubano.

Los cubanos, junto con los inmigrantes del Este, es el grupo que más solicita asilo, y uno de los que más busca la nacionalización: muchos de ellos se consideran españoles nacidos en Cuba. La mayoría de las características singulares de la migración de cubanos hacia España hunden sus raíces en la historia de los dos países.

La pirámide de edades de la comunidad de inmigrantes cubanos antes de la última década del siglo XX presentaba claros rasgos de envejecimiento. El porcentaje de personas de más de 65 años era mayor que los de otras comunidades de inmigrantes, como las de los europeos occidentales, que eran, entre los extranjeros, los más envejecidos, contrastando con los procedentes de otros países hispanoamericanos, con los que tienen evidente relación y que, en cambio, eran mucho más jóvenes. Esa elevada proporción de ancianos explica también la numerosa presencia de viudos: casi 15 por ciento (IDEM). Si a ello sumamos la escasez de niños, sobre todo en 
los primeros años de vida, los rasgos de envejecimiento de este grupo eran todavía más acentuados que los de cualquier otro colectivo de extranjeros. Es importante señalar que los jóvenes asentados en España en su mayoría se encontraban en el rango de edad entre los 25-34 años.

El nivel de estudios era elevado, predominando los que tienen estudios superiores. Los asentados en esta última etapa del siglo XX se distribuían por las comunidades autónomas españolas, con una clara concentración en la comunidad de Madrid y en las comunidades de las que procedían la mayor parte de los emigrantes que fueron a Cuba en el pasado: Canarias, Asturias y Galicia. Dentro de éstas, se instalaban preferentemente en las capitales provinciales y en ciudades de más de 100 mil habitantes.

El nuevo grupo de inmigrantes cubanos que comienza a llegar a España en la última década del siglo XX e inicios del siglo XXI se caracteriza por la estructura siguiente. Un predominio de mujeres (57.4 por ciento) y de adultos, de entre 25 y 40 años. Su distribución espacial es por todo el territorio, sin embargo, siguen mostrando interés por las regiones de las que salieron en el pasado, los españoles hacia Cuba. Las comunidades con más inmigrantes cubanos a principio del siglo XXI, son Canarias con 5,568 personas seguida de Madrid, con 5,398, y Cataluña, con 3,607 y la Comunidad Valenciana, con 2,013 (INE, 2012). Como se representa en la Figura 2.

Figura 2: Principales núcleos de asentamientos de inmigrantes cubanos en España en el año 2001

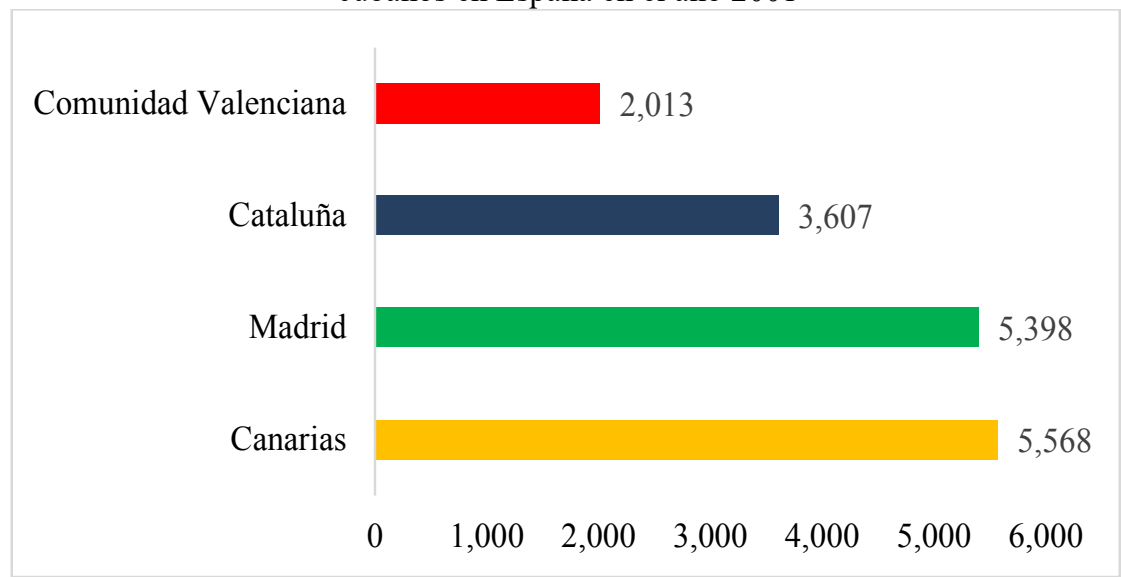

Fuente: elaboración propia a partir de los datos tomados del INE. 
Los cubanos siguen siendo de los grupos que en mayor proporción solicitan asilo político, dadas las circunstancias que concurren en su país. En 2002 fueron la segunda nacionalidad solicitante de asilo, con 18.7 por ciento, detrás de Nigeria, aunque a partir de 2003, ha disminuido notablemente el número de solicitantes respecto a años anteriores. En cambio, las nacionalizaciones siguen siendo muy numerosas.

A pesar de que cada vez son más similares a los restantes inmigrantes económicos, los cubanos mantienen, como los argentinos, un mayor nivel cultural, como lo demuestra el que sea uno de los colectivos que en mayor medida solicita la homologación de títulos universitarios con 463 solicitudes en 2003, sólo superadas por las de Argentina, Colombia, Italia y Perú. Una parte son estudiantes con autorización de estancia con 232 estudiantes en 2003 (en este caso con predominio de varones, con un 58 por ciento). También son relativamente numerosos los que se encuentran realizando estudios universitarios de segundo y tercer ciclo. Igualmente, es un indicador de la superioridad en la formación el hecho de que en el régimen de cotización a la seguridad social 9.3 por ciento de los cubanos registrados sean ingenieros y licenciados.

A comienzos de 2004, 54 por ciento de los cubanos residentes en España se encontraban en situación laboral activa, la mayoría trabajando por cuenta ajena y ocho por ciento por cuenta propia, en los diferentes regímenes de la Seguridad Social. En el régimen general y con contratos indefinidos, en 40 por ciento, pero también en algunos de los regímenes especiales, como el de empleadas de hogar, que acoge a 3.24 por ciento de los trabajadores cubanos.

En los primeros años de la llegada de cubanos a España era raro que se ocuparan en estos trabajos. Incluso, tal como veíamos en la primera parte, hasta 1991 había, entre los cubanos, un predominio de profesionales, técnicos y directivos, 25 por ciento, frente a 4.6 por ciento de obreros sin especializar. Actualmente, en cambio, el porcentaje de peones alcanza 23.02 por ciento, mientras que sólo son directivos y técnicos en torno a 12 por ciento.

\section{Conclusiones}

Todo ello confirma que el colectivo de cubanos cada vez tiene más carácter de migración económica, frente a su anterior situación más relacionada con circunstancias políticas. De la comparación de los dos momentos censales, 1991-2001, se desprenden algunos notables cambios en las características de la inmigración cubana, a pesar de que la situación de origen no ha varia- 
do en lo fundamental. Sí lo han hecho, en cambio, determinadas circunstancias que han afectado a la economía cubana como la caída del campo socialista, el recrudecimiento del bloqueo norteamericano y las ineficiencias asociadas al modelo de crecimiento económico, que ha afectado considerablemente la sociedad cubana sumiéndola en una situación de crisis que se ha acrecentado a lo largo de estos primeros años del siglo XXI.

Como muestran datos aportados por el INE, desde el año 1998 hasta 2011 el volumen de cubanos que arribó a España ha ido en aumento, pasando de los 10469 que había en el año 1998 a 54,680 en el año 2011.

Otra de las características de los nuevos inmigrantes cubanos es la presencia de un grupo significativo que ha optado para establecerse en España por la vía del matrimonio con ciudadanos españoles, lo cual les permite, si regresan cada año a Cuba, no perder sus derechos como ciudadanos cubanos. Por otro lado, hay un grupo menos significativo, pero importante porque la mayoría se encuentra en situación irregular, que viajan a España, en calidad de viaje oficial, para realizar cursos de formación (en este caso, incluso, por parte de algunas de las empresas españolas asentadas en Cuba como hoteles y bancos) y que una, vez en España, deciden quedarse.

Asimismo, la presencia de cubanos en muchos países de Europa, dadas las antiguas relaciones de Cuba con los países del Este, facilita el envío de cartas de invitación, vía muy utilizada para salir del país. En este sentido, es importante señalar que un buen número de los que por este método acceden a países europeos tratan luego de entrar en España. Además, es incluso frecuente que, si antes de llegar al país del que procede la carta de invitación, el vuelo realiza escala en España aprovechan y se quedan como inmigrantes económicos en situación irregular.

Hasta el 1 de enero de 2011 los principales núcleos de asentamientos de inmigrantes cubanos en España no habían variado, pero si había aumentado su población. Otra de las características, de un grupo significativo, de los emigrados cubanos es que no ven a España como el punto final del proceso migratorio, sino como un país puente para su destino final: los Estados Unidos de América.

Puede afirmarse que una de las características significativas de la migración cubana hacia España, a principio del siglo XXI, es la tendencia a la feminización de la inmigración, la presencia de una población inmigrante con un alto nivel educativo y un cambio en el perfil de los inmigrantes, que ya no son tantos disidentes políticos, sino, sobre todo, inmigrantes económicos, que quieren buscar oportunidades en España, para mejorar su situación personal y ayudar a sus familiares en Cuba. 


\section{REFERENCIAS BIBLIOGRÁFICAS}

Aja Díaz, Antonio, 2002, Tendencias y retos de Cuba ante el tema de la emigración. CEMI. Universidad de la Habana, Cuba.

Basch, L., Glick-Schiller, N. y Szanton Blanc, C., 1994, Nations Unbound. Transnational Projects, Postcolonial Predicaments and Deterritorialized Nation-States. USA: Gordon and Breach Science Publishers.

Click, N., Basch, L., Szanton, C., 1995, "From Immigrant to Transmigrant”, Theorizing Transnational Migration, vol. 68, núm. 1, pp. 48-63, Published by: The George Washington University Institute for Ethnographic Research Stable URL: http://www.jstor.org/stable/3317464 Accessed: 11/11/2010 16:59

García Ferrando, Manuel, 1996, El Análisis de la realidad social. Métodos y técnicas de investigación. Alianza Editorial, S. A. Madrid, España.

García Moreno, C., 2009, "No es fácil... Y aquí tampoco. Trayectorias migratorias de mujeres cubanas en España". Actas del VI Congreso sobre las migraciones en España. A Coruña. España.

García Moreno, C., 2012, Mujeres migrantes cubanas. Resolviendo e inventando también en España. Tesis doctoral. Universidad Rovira i Virgili, Tarragona, España.

Goñalons, Pilar, Flecha, Ramón, Santa Cruz, Iñaki y Gómez, Carlos, 2008, "Las aportaciones y los retos de la perspectiva transnacional: Una lectura de género", en VVAA, Nuevos retos del transnacionalismo en el estudio de las migraciones. Barcelona. Disponible en http://docsgedime.wordpress.com

González Yanci, María Pilar y Aguilera Arilla, María, 2002, La inmigración cubana en España. Razones políticas y de sangre en la elección de destino. espacio, tiempo y forma. Serie VI, Geografía, Tomo 15, UNED, España.

Gregorio, C., 1998, Migración femenina. Su impacto en las relaciones de género, Madrid: Narcea. España.

Guarnizo Guarnizo, Luis Eduardo, 2004, “Aspectos económicos del vivir transnacional”, en Escriva, Ángeles y Ribas, Natalia (coords.), Migración y desarrollo. Estudios sobre remesas y otras prácticas transnacionales en España, Córdoba, CSIC.

Herrera, G., 2005, "Mujeres ecuatorianas en las cadenas globales del cuidado", en Herrera, G., Carrillo, M.C. y Torres, A., La migración ecuatoriana. Transnacionalismo, redes e identidades. FLACSO, Plan Migración, Comunicación y Desarrollo. Quito.

INE, 2009, Padrón de extranjeros en España en el año 2009, Instituto Nacional de Estadística (INE). Madrid, España: disponible en http://www.ine.es. Consultado el $11 / 10 / 2016$.

INE, 2012, Anuario Estadístico de España 2012, Instituto Nacional de Estadística (INE) disponible en http://www.ine.es. Consultado el 28/09/2016. Madrid. 
Itzigsohn, J. y Saucedo, S., 2002, “Immigrant Incorporation and Sociocultural Transnationalism”, en International Migration Review, núm. 36.

Itzigsohn, J., 2000, "Immigration and the Boundaries of Citizenship: The Institutions of Immigrants' Political Transnationalism", en International Migration Review, núm. 34, pp. 1126-1155.

Itzigsohn, J., Dore, C., Medina, E., y Vázquez, O., 1999, “Mapping Dominican Transnationalism: Narrow and Broad Transnational Practices", en Ethnic and Racial Studies, núm. 22, pp. 2316-2340.

Lehmann, E. L., 1986, Testing Statistical Hypotheses, Wiley.

Luis Sosa, Jorge, 2012, El proceso migratorio cubano entre la flexibilidad y la integración. Vivir lo transnacional en España a inicios del siglo XXI. Tesis doctoral. Universidad de Zaragoza, España.

Martín Consuelo, 2000, Emigración y vida cotidiana en Cuba, Tesis Doctoral. Universidad de la Habana, Cuba. Disponible en http://www.uh.cu/centros/cemi/ wpontent/uploads/2011/11/9MigraCubaEspana.pdf. Consultado el 2/07/2016.

Massey Douglas, S., 1998, Worlds in motion. Understanding International Migration at the End of the Millennium. Clarendon Press.

Ministerio de Empleo y Seguridad Social, 2008, Observatorio Permanente de la Inmigración, Madrid, España.

ONEI, 2017, Anuario Estadístico de Cuba 2009, ONEI, disponible en http://www. one.cu. Consultado el 18 noviembre de 2018. La Habana.

Oso, L., 1998, La migración hacia España de mujeres jefas del hogar. Instituto de la Mujer. Madrid, España.

Pajares, M., 2008, Inmigración y mercado de trabajo. Informe 2008, Madrid, Ministerio de Trabajo y Asuntos Sociales, Documentos del Observatorio Permanente de la Inmigración, 17.

Parreñas, R., 2001, The Global Servants: Migrant Filipinas Domestic Workers in Rome and Los Angeles, Palo Alto: Standford University Press.

Pedone, C., 2005, Tu siempre jalas a los tuyos. Cadenas y redes migratorias de las familias ecuatorianas hacia España, en Herrera, G., Carrillo, M.C. y Torres, A. (eds.) La migración ecuatoriana. Transnacionalismo, redes e identidades. Quito, FLACSO-Plan Migración Comunicación y Desarrollo, pp. 105-146.

Portes, A., 1996b, "Global villagers: the rise of transnational communities", en The American Prospect, vol. 25, pp. 74-77.

Portes, A., 1996a, "Transnational Communities: their emergence and significance in the contemporary world-system", en Korzeniewidcz, R.P. y Smith, W.C. (comps.), Latin America in the World Economy, Westport, CT: Greenwood Press, pp. 151-168.

Portes, A., 2005, “Convergencias teóricas y evidencias empíricas en el estudio del transnacionalismo de los inmigrantes”, en Migración y Desarrollo, núm. 4, primer 
semestre, 2005, pp. 2-19 Red Internacional de Migración y Desarrollo Zacatecas, México.

Portes, Alejandro, y Bach, Robert L., 1985, Latin Journey: Cuban and Mexican Immigrants in the United States, Berkeley, CA:University of California Press.

Riaño, Y. y Richter, M., 2008, "Cuáles podrían ser enfoques conceptuales apropiados para el estudio de las prácticas transnacionales de los migrantes. El potencial de las perspectivas de "espacio social transnacional y de la interseccionalidad de clase, etnicidad y género". Ponencia presentada en el Simposio internacional Nuevos retos del transnacionalismo en el estudio de las migraciones, Barcelona, 14-15 de febrero de 2008.

Roca, J., Monstserrat, G., Bodoque, Y., 2008, Migraciones por amor: diversidad y complejidad de las migraciones de mujeres, Universitat Rovira i Virgili. Departament d'Antropologia, Filosofia i Treball.

Sassen, Saskia, 1998, Globalization and Its Discontents, The New Press, Nueva York.

Suárez, L., 2004, "Transformaciones de género en el campo transnacional. El caso de las mujeres inmigrantes en España”, en La Ventana, núm. 20, pp. 293-331

Valles, S. Miguel, 1999, Técnicas cualitativas de investigación social. Reflexión metodológica y práctica profesional. Editorial Síntesis, Madrid, España

\section{RESUMEN CURRICULAR DE LOS AUTORES}

\section{Jorge Luis Sosa}

Licenciado en Sociología por la Universidad de la Habana, Cuba, y profesor del Departamento de Sociología de la Universidad Marta Abreu de las Villas, Cuba desde el 2003 al 2010. Maestro en Gestión y Dirección del Bienestar Social y los Servicios Sociales por la Universidad de Alcalá, España, Maestro Universitario en Cooperación al Desarrollo por la Universidad de Valencia, España, y Doctor en Sociología de las Políticas Públicas y Sociales, Universidad de Zaragoza, España, Premio Cum Laude. Actualmente es asesor de la Fundación Cronos Vida y Cultura, España y Profesor Titular e Investigador de la Universidad Autónoma de Ciudad Juárez. Entre sus publicaciones más importantes destacan: Los Equilibrios del Tercer Sector. Una Filosofía del Pluralismo de Funciones. Gestión de Procesos Sociales. La Experiencia de Cuba, España y México. Mirada Sociológica al Proceso Migratorio Cubano en los Albores del Siglo XXI. África un Continente que Camina. Miradas Migratorias Regionales en México. Análisis, Perspectivas y Propuestas de Intervención Social.

Orcid: 000-0002-7604-6430

Dirección electrónica: jorge.luis@uacj.mx 
Addiel Pérez Díaz

Licenciado y Maestro en Sociología por la Universidad de la Habana y Doctor en Ciencias Sociológicas por la Universidad Centra "Marta Abreu" de las Villas (Cuba). Ha sido vicedecano de investigación y postgrado de la facultad de Ciencias Sociales en la Universidad Central "Marta Abreu" de las Villas; coordinador e investigador de varios proyectos en temáticas como el desarrollo local, gobiernos locales, desarrollo comunitario y estudios de población. Profesor invitado y becario en diversas universidades españolas. Profesor de la Licenciatura en Sociología, maestría y doctorado en Desarrollo Comunitario en la Universidad Central de las Villas, donde trabajó de 2001 al 2013. Profesor de la Maestría en Sociología de la Universidad de la Habana. De 2013 a la actualidad es Profesor de Tiempo Completo, adscrito al departamento de Ciencias Sociales, en la Universidad Autónoma de Ciudad Juárez. Es miembro del Sistema Nacional de Investigadores. Ha escrito ocho artículos en revistas indexadas, y más de una veintena, entre libros y capítulos sobre las temáticas anteriormente enunciadas.

Orcid: 000-0002-1875-8384

Dirección electrónica: addiel.perez@uacj.mx

Artículo recibido el 20 de septiembre de 2016 y aprobado el 11 de septiembre de 2018. 